\title{
Treatment with Glimepiride, but not Mitiglinide and Short-Acting Insulin, Resists Body Weight and Abdominal Fat Reduction Under Dietary Energy Restriction
}

\author{
Takanari Nakano ${ }^{1,5}$, Ikuo Inoue ${ }^{2}$, Kentaro Satoh², Mochihito Yamazaki ${ }^{3}$, Takuya Awata², Susumu Kurihara², \\ Sei-ichi Goto ${ }^{4}$, Yu-ichi Shinoda ${ }^{1}$, Tsugikazu Komoda ${ }^{1}$, and Shigehiro Katayama ${ }^{2}$ \\ ${ }^{1}$ Department of Biochemistry, Faculty of Medicine, Saitama Medical University, Saitama, Japan \\ ${ }^{2}$ Diabetes and Endocrinology, Faculty of Medicine, Saitama Medical University, Saitama, Japan \\ ${ }^{3}$ Kumagaya Surgical Hospital, Saitama, Japan \\ ${ }^{4}$ Division of Internal Medicine, Department of Comprehensive Medical Sciences, Meikai University School of Dentistry, Saitama \\ Japan \\ ${ }^{5}$ Department of Medicine, School of Medicine, University of California Los Angeles
}

JAtheroscler Thromb, 2009; 16:63-66.

Key words; Obesity, Lifestyle intervention, Oral hypoglycemic agents, Type 2 diabetes mellitus, Syndrome of inappropriate insulin secretion (SIIS)

Postprandial hyperglycemia plays a key role in the onset and development of type 2 diabetes mellitus ${ }^{1)}$, but sulfonylureas (SUs), a popular choice for oral hypoglycemic agents, are much less robust in the prandial period $^{2)}$ and lead to unwanted body weight gain ${ }^{3)}$. Glimepiride, a third-generation SU, has a lower risk of hypoglycemia and less body weight gain ${ }^{4,5)}$; however, it shows insufficient restoration of mealtime insulin secretion $^{6}$. Mitiglinide, a newly developed short-acting insulin secretagogue with favorable pharmacological efficacy over the popular nateglinide ${ }^{7,8)}$, restores prandial insulin secretion ${ }^{9)}$, and prevents secondary pancreatic $\beta$-cell failure, hypoglycemia, and hyperinsulinemia ${ }^{3,8,10}$. The switch from SUs to glinides seems to be beneficial for patients with type 2 diabetes mellitus or prediabetic conditions; however, it has been considered to lead to insufficient glycemic control.

Successful lifestyle intervention results in good glycemic control by improving insulin activity and pancreatic $\beta$-cell response to insulin secretory stimuli $^{11,12)}$. Thus, in combination with lifestyle intervention, switching from SUs to glinides is likely to be feasible, and SUs might be unnecessary in these patients.

Address for correspondence: Takanari Nakano, Bldg. 114, Suite 217, West Los Angeles VA Medical Center, 11301 Wilshire

Blvd., Los Angeles, CA 90073 USA

E-mail: nk.takanari@gmail.com

Received: April 8, 2007

Accepted for publication: September 25, 2008
We report here that glimepiride could be switched to mitiglinide under calorie restriction conditions, and that treatment with glimepiride, but not mitiglinide and rapid-acting insulin, resisted body weight and abdominal fat reduction in diabetic patients under dietary calorie restriction.

Fig. 1 shows the design of this prospective and randomized meal replacement study and the patient group analyzed. Table 1 shows the demographic characteristics of the subjects at baseline. Study measurements were performed in the clinical chemistry laboratories of our hospitals. Computed tomography (CT) scans of the abdomen were acquired using an electron beam CT scanner (TCT-900S Helix; Toshiba, Tokyo, Japan). Values are the mean \pm SE. Differences between mean values before and after the test period were assessed with the paired $t$-test. For multiple group comparisons, homogeneity of variance was assessed by the Levene test. Student's $t$-test was used for parametric comparisons. When the Levene test revealed significant differences between groups, the Kruskal-Wallis test was performed on the variables. We considered $p$ values $<0.05$ as significant. All analyses were performed using $\mathrm{JMP}^{\circledR}$ version 6.0 (SAS Institute).

In the meal replacement study with 229 diabetic patients, MicroDiet ${ }^{\circledR}$ (MD) treatment reduced their body weight by $4.7 \%$. In the 17 subjects analyzed in the present study (see Fig. 1), the body weight decrease was greater $\left(-7.7 \%\right.$ ) (Table 2). Mean $\mathrm{HbA}_{1 \mathrm{C}}$ 


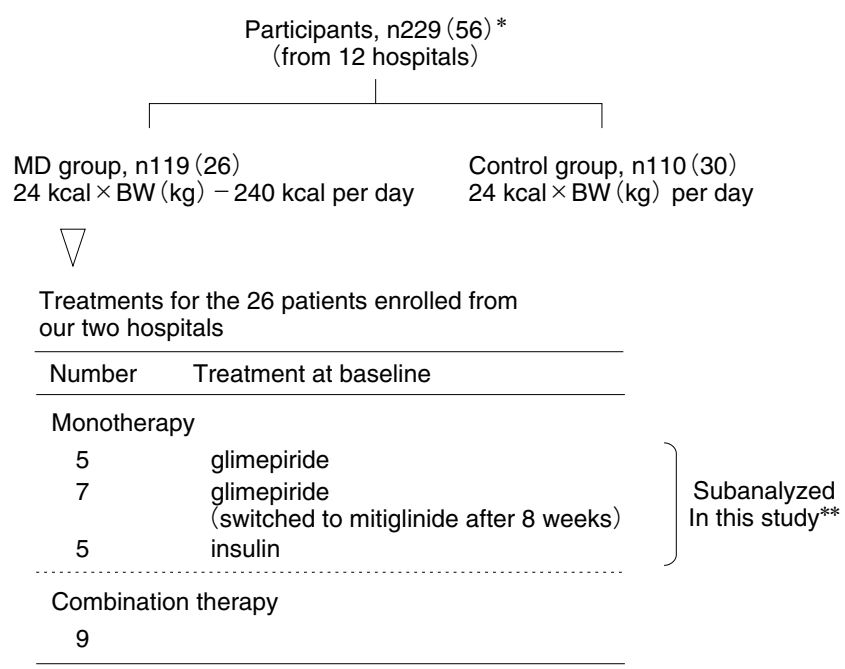

Fig. 1. Overview of a meal replacement study.

A prospective and randomized meal replacement study was conducted in Japan in 2006 of 229 patients with impaired glucose tolerance or type 2 diabetes mellitus with the collaboration of 12 hospitals, to examine whether calorie restriction for 24 weeks reduced body weight and ameliorated risk factors for cardiovascular disease (Fig. 1). The calorie restriction group replaced one meal per day (of their choice) with a low-calorie formula diet, MicroDiet ${ }^{\circledR}$ (MD, $240 \mathrm{kcal}$ per meal, Sunny Health, Japan), which consists of shakes and crackers. Briefly, the criteria for enrollment were as follows: patients with a diagnosis of impaired glucose tolerance or type 2 diabetes mellitus as defined by the World Health Organization criteria who were regularly visiting hospitals, 20 to 70 years of age, and with a body mass index (BMI) of $\geq 25 \mathrm{~kg} / \mathrm{m}^{2}$. Criteria for exclusion were concomitant treatment of chronic diseases, including renal, liver, and cardiovascular diseases, recent acute illness, or change in diet, treatment, or lifestyle within the 3-month period preceding the study.

Of the 56 patients who attended our hospitals, 26 participants were assigned to the MD group. Among them, 17 patients were treated with monotherapy (glimepiride, $n=12$, and insulin, $n=5$ ) and 9 with combination therapy using pioglitazone, acarbose, glimepiride, and metformin at baseline. Regular clinical analyses showed that the 12 patients with glimepiride monotherapy had $<8 \% \mathrm{HbA}_{1 \mathrm{c}}$ and $<8 \mathrm{mmol} / \mathrm{L}$ fasting blood glucose level. Of these 12 patients, 7 agreed to switch medication to mitiglinide under careful monitoring of the fasting blood glucose level (mitiglinide group). The remaining 5 patients continued to receive glimepiride (glimepiride group). We also analyzed findings for five patients treated with rapid-acting insulin monotherapy InnoLet ${ }^{\circledR}$, as an insulin-treated group (insulin group).

*, Numbers of patients enrolled from our two hospitals (Saitama Medical University and Kumagaya Surgical Hospital) are indicated in parentheses. ${ }^{* *}$, The 17 patients treated with glimepiride or insulin monotherapy were subanalyzed in the present study.

level decreased by $1.0 \%$. Other metabolic parameters, including lipids and blood pressure, were not significantly altered.

Eight weeks after the start of the meal replacement study, the 12 patients treated with glimepiride improved glycemic control $\left(\mathrm{Hb} A_{1 \mathrm{c}},<8 \%\right.$; fasting blood glucose level, $<8 \mathrm{mmol} / \mathrm{L}$ ) and, among them, seven patients switched from glimepiride to mitiglinide. There was no deterioration of glycemic control during the study period. $\mathrm{Hb} \mathrm{A}_{1 \mathrm{C}}$ value decreased in the two groups $(-1.4 \%$ in the mitiglinide group and $-1.2 \%$ in the glimepiride group), while IRI level decreased only in the mitiglinide group $(-27 \mathrm{pmol} / \mathrm{L}$ compared to before the study, $p<0.001)$. BMI was reduced in the mitiglinide $\left(-3.0 \mathrm{~kg} / \mathrm{m}^{2}, p<0.0001\right)$ and the low-dose insulin-treated groups $\left(-2.2 \mathrm{~kg} / \mathrm{m}^{2}\right.$, $p=0.004)$, but not in the glimepiride group $(-1.5$ $\left.\mathrm{kg} / \mathrm{m}^{2}, p=0.68\right)$ after 6 -month treatment with MD (Table 2). The abdominal fat area decreased by approximately $20 \%$ in the mitiglinide group and by $24 \%$ in the low-dose insulin group.

The present results showed that glimepiride could be replaced by mitiglinide under calorie restriction intervention. We also found that the glimepiride group resisted the reduction of body weight and abdominal fat with calorie restriction, while patients who switched from glimepiride to mitiglinide (mitiglinide group) and patients on rapid-acting insulin therapy (insulin group) exhibited larger reductions in these parameters. Moreover, we observed a significant greater decrease in body weight, waist, and BMI in the mitiglinide group than in the glimepiride group.

Although some clinicians expected glinides to be useful alternative to SUs with the advent of new insulin secretagogues, attempts to use them often led to deterioration of glycemic control, probably resulting from lower insulin secretion by glinides. In the present study, calorie restriction decreased the need for glimepiride by improving insulin resistance and consequently, fasting hyperglycemia, and thus it appeared to be a key to such achievement with mitiglinide. The meal replacement study showed that MD treatment reduced the mean fasting blood glucose and $\mathrm{HbA}_{1 \mathrm{C}}$ level by approximately 5\% (MD group, $n=119$ in 12 hospitals), and these levels decreased over 20 weeks ${ }^{13)}$.

Unbalance between insulin secretion and blood glucose concentration underlies the development of metabolic abnormalities. The early phase of type 2 diabetes mellitus can be seen in part as a disorder of appropriate insulin secretion, that is, late, weak, or both types of insulin secretion in response to meals. We have named such conditions "Syndrome of inappropriate insulin secretion (SIIS)" and believe that SIIS is a crucial condition in the development of diseases associated with metabolic abnormalities, including metabolic syndrome. We speculate that naturalizing the pattern of insulin secretion with medications is a key to prevent and/or slow its onset or disease devel- 
Table 1. Patient characteristics at baseline

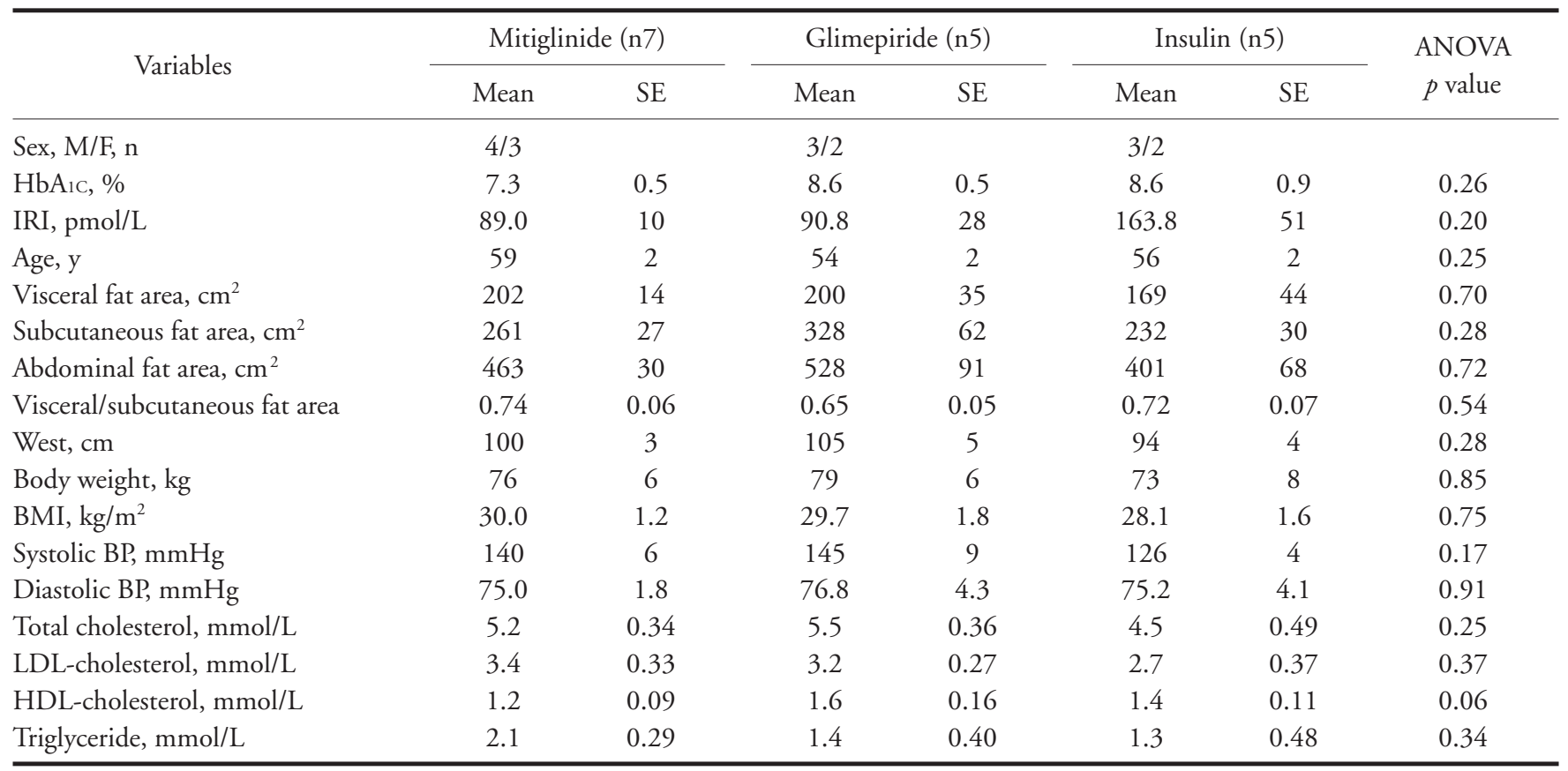

Table 2. Changes in clinical parameters after 24-week calorie restriction

\begin{tabular}{|c|c|c|c|c|c|c|c|c|}
\hline \multirow{2}{*}{ variables } & \multicolumn{8}{|c|}{ Group } \\
\hline & change (\%) & $p$ value & change (\%) & $p$ value & change (\%) & $p$ value & change (\%) & $p$ value \\
\hline visceral fat area & -18 & 0.001 & -20 & 0.01 & -7 & 0.18 & $-24^{*}$ & 0.02 \\
\hline subcutaneous fat area & -16 & $<0.0001$ & $-27^{*}$ & $<0.0001$ & -5 & 0.30 & -18 & 0.04 \\
\hline visceral/subcutaneous fat area & -2.5 & 0.7 & -19.0 & 0.25 & 1.1 & 0.90 & 1.5 & 0.78 \\
\hline BW & -7.7 & $<0.0001$ & $-10.0^{*}$ & 0.0001 & -4.6 & 0.07 & -8.0 & 0.01 \\
\hline BMI & -7.7 & $<0.0001$ & $-10.0^{*}$ & $<0.0001$ & -4.6 & 0.68 & -8.0 & 0.004 \\
\hline Abdominal fat area/BMI & -10.0 & 0.0005 & $-14.5^{* *}$ & 0.002 & 1.8 & 0.67 & $-14.0 *$ & 0.04 \\
\hline $\mathrm{HbA}_{1 \mathrm{c}}$ & -12 & $<0.0001$ & -19 & 0.04 & -14 & $<0.001$ & -10 & 0.004 \\
\hline IRI & -18.0 & 0.06 & -30.0 & 0.001 & 0.1 & 0.64 & -15.0 & 0.46 \\
\hline HDL-cholesterol & -1.5 & 0.98 & 3.9 & 0.42 & -3.2 & 0.58 & 7.4 & 0.23 \\
\hline Triglyceride & -13 & 0.02 & -29 & 0.06 & -2 & 0.42 & -15 & 0.24 \\
\hline
\end{tabular}

${ }_{p} p 0.05$ and ${ }^{* *} p<0.01$ versus glimepiride

opment.

The present study has three major limitations. First, the number of patients enrolled was small, risking bias. A randomized, prospective study with type 2 diabetic patients is now ongoing to confirm our find- ings, and preliminarily results have shown that the combination of calorie restriction and mitiglinide has effects similar to those observed in the present study (data not shown). Next, although body weight reduction by lifestyle intervention was successful in the set- 
ting of an interventional clinical trial (body weight reduction $-7.7 \mathrm{~kg}$ ), patients often failed to continue lifestyle changes in non-clinical trial settings. Patients and clinicians should therefore aim to maintain calorie restriction. Finally, SUs increase appetite during the inter-meal period, which might make it difficult for patients in SU groups to follow the instructions for calorie restriction. The unchanged abdominal fat area and BMI in the glimepiride group might be due to compliance difficulties.

In conclusion, the present study showed that the combination of calorie restriction and mitiglinide lowered the abdominal fat area and body weight, whereas glimepiride reduced neither significantly. These findings suggest that combined use of mitiglinide with calorie restriction is warranted, and that short-acting oral hyperglycemic agents and exogenous short-acting insulin are useful for those receiving instruction in lifestyle change.

\section{Acknowledgements}

The meal replacement study in 2006 in Japan was supported by Sunny Health (Nagano, Japan). The authors thank Rina Shinozaki, Sawako Satoh, and Yuka Nakano for secretarial assistance.

\section{References}

1) Polonsky KS, Given BD, Hirsch LJ, Tillil H, Shapiro ET, Beebe C, Frank BH, Galloway JA, Van Cauter E: Abnormal patterns of insulin secretion in non-insulin-dependent diabetes mellitus. N Engl J Med, 1988; 318:12311239

2) Carroll MF, Izard A, Riboni K, Burge MR, Schade DS: Control of postprandial hyperglycemia: optimal use of short-acting insulin secretagogues. Diabetes Care, 2002; 25:2147-2152
3) Krentz AJ, Bailey CJ: Oral antidiabetic agents: current role in type 2 diabetes mellitus. Drugs, 2005; 65:385-411

4) Massi-Benedetti M: Glimepiride in type 2 diabetes mellitus: a review of the worldwide therapeutic experience. Clin Ther, 2003; 25:799-816

5) Gottschalk M, Danne T, Vlajnic A, Cara JF: Glimepiride versus metformin as monotherapy in pediatric patients with type 2 diabetes: a randomized, single-blind comparative study. Diabetes Care, 2007; 30:790-794

6) Geisen K: Special pharmacology of the new sulfonylurea glimepiride. Arzneimittelforschung, 1988; 38:1120-1130

7) Sunaga Y, Gonoi T, Shibasaki T, Ichikawa K, Kusama H, Yano H, Seino S: The effects of mitiglinide (KAD-1229), a new anti-diabetic drug, on ATP-sensitive $\mathrm{K}^{+}$channels and insulin secretion: comparison with the sulfonylureas and nateglinide. Eur J Pharmacol, 2001; 431:119-125

8) Shigeto M, Katsura M, Matsuda M, Ohkuma S, Kaku K: Nateglinide and mitiglinide, but not sulfonylureas, induce insulin secretion through a mechanism mediated by calcium release from endoplasmic reticulum. J Pharmacol Exp Ther, 2007; 322:1-7

9) Ichikawa $K$, Yamato $T$, Ojima $K$, Tsuji A, Ishikawa $K$, Kusama H, Kojima M: Effect of KAD-1229, a novel hypoglycaemic agent, on plasma glucose levels after meal load in type 2 diabetic rats. Clin Exp Pharmacol Physiol, 2002; 29:423-427

10) Ojima K, Ichikawa K, Fujimori Y, Aoyagi I, Yamato T, Tsuji A, Kusama H, Kojima M, Shibata N: Rapid onset-insulinotropic effect of mitiglinide calcium dihydrate (KAD-1229), a novel antipostprandial hyperglycemic agent. Jpn Pharmacol Ther, 2004; 32:73-80

11) Albu J, Raja-Khan N: The management of the obese diabetic patient. Prim Care, 2003; 30:465-491

12) Saito Y, Ishikawa Y, Shinomiya M, Shirai K, Yoshida S: Effects of protein-sparing modified fasting on obese Japanese patients. J Clin Biochem Nutr, 1987; 2:91-100

13) Saito Y, Shirai K, Oikawa S, Teramoto T, Yamada N, Ishibashi S, Tada N, Miyazaki S: Effectiveness of MicroDiet and ordinary meals on the improvement of glucose and lipid metabolism per unit weight reduction in obese diabetic patients. In 19th World Diabetes Congress Cape town, South Africa, 2006 